\title{
Spending on BSE research
}

SIR - As one of the industry-independent representatives on the Government's Priorities Board for Research and Development (R\&D) in Food and Agriculture from 1990 to 1994 , I chaired the Ruminants Advisory Sectoral Group (RASG). The group advised the government through the priorities board on $R \& D$ in the various areas in the ruminant sector, which included animal welfare and disease, and had access to information on government and other research projects and their associated funding.

It is in that previous research role that I am responding to the article on bovine spongiform encephalopathy (BSE) (Nature 382, 755-756; 1996) by David Skegg. His article accompanied the publication of the work of Anderson et al. (382, 779-788; 1996). Although Skegg summarizes the situation as accurately as is possible in the light of the long-term nature and complexities of the disease and associated $\mathrm{R} \& \mathrm{D}$, the statement in his final paragraph about spending on BSE research is inaccurate and misleading.

Skegg states that "during the three years 1988 to 1991 the UK government invested the paltry sum of $£ 3$ million on BSE-related research". There have always been several sources of funding for government BSE research, and it would appear that Skegg has not had access to all the information relating to BSE R\&D and its funding for 1988 to 1991. The information collated by the RASG in 1991 indicated annual expenditure from all sources of considerably more than $£ 3$ million. The RASG report for 1991-92 shows that, of the total spent on the ruminant welfare and disease area of $£ 20.134$ million, £8.324 million was for BSE and related scrapie work.

It is interesting that the BSE R\&D expenditure was some $20 \%$ of the total covered by the ruminant group, which included work on forages, nutrition, reproduction and genetics, meat and milk production, and production systems, as well as other disease and animal welfare issues.

The Ministry of Agriculture, Fisheries and Food (MAFF) document Research Strategy 1996-2000 shows its expenditure on BSE research for $1996-97$ to be $£ 10.400$ million. That is some $66 \%$ of the total MAFF expenditure of $£ 15.858$ million in the animal health and welfare sector. This is complemented by further R\&D by other sponsors such as the Biotechnology and Biological Sciences Research Council, the Medical Research Council and the Wellcome Trust.

lan Howie

Ryfield,

Wormbridge,

Hereford HR2 9DB, UK

SIR - The British and European Union (EU) authorities were recently accused by
D. W. van Bekkum and P. J. Heidt (Nature 382, 574; 1996) of having missed an early opportunity of resolving the issue of the risk to humans from bovine spongiform encephalopathy (BSE) by their alleged failure to initiate and fund challenge experiments in chimpanzees available in Europe.

There were, however, discussions involving UK and EU scientists and authorities in 1989 and 1990 about the feasibility of treating chimpanzees with material from BSE-infected cows. As a virologist and deputy director of the Dutch primate centre at that time, I was directly involved in some of those discussions.

It was impossible to design conclusive study protocols answering the questions of human infectivity of the BSE agent and of the risk for the consumer of beef products, which are different issues. To show infectivity, the intracranial injection of infected brain extracts was considered. To evaluate the risk to consumers, the only relevant route is oral challenge with beef from infected animals. From epidemiological studies, it is clear that if BSE is causing disease in humans, the transmission rate is very low, needing hundreds of chimpanzees to get significant results, more than the number available in Europe.

But even if oral transmission had resulted in disease in chimpanzees, the relevance of these results for humans could be argued. It is doubtful whether chimpanzees and humans have similar sensitivities to pathogens transmitted by meat. Chimpanzees are almost exclusively vegetarian, and resistance in humans to the BSE agent may have been selected for by hundreds of thousands of years of meat consumption. At the other extreme, if animals injected with heavily contaminated material in their brains remained healthy, the relevance for humans could again have been challenged.

Although van Bekkum and Heidt state otherwise, there is no evidence that chimpanzees are the experimental animal of choice to attack this problem. Knowledge of the pathogenesis of natural infections in chimpanzees is limited, and there is experience only with a few human pathogens in this species. The most widely studied have been the hepatitis viruses and human immunodeficiency virus (HIV). And although chimpanzees have been instrumental in testing vaccines for hepatitis-B virus, the course of acute hepatitis and especially the long-term sequelae of chronic infections, which are the most relevant for the human disease, are mild or absent in chimpanzees. With two exceptions, the hundred or so chimpanzees infected with HIV failed to develop symptoms similar to human AIDS. So any negative results with BSE in chimpanzees would be difficult to extrapolate to safety of beef from BSE-infected animals for human consumption.

In summary, the British and EU authorities considered chimpanzee experiments at an early stage of the BSE epidemic and have consulted the relevant specialists in the field. Both DGXII of the European Commission (the science directorate) and the UK government have from the beginning of the epidemic funded research into the BSE problem, and both were willing to provide the vast amounts of money that the chimpanzee experiments would have cost.

The many unanswered questions about BSE (and prions in general) have less to do with the lack of funding or the handling of the problem by the British or EU authorities than with the fact that BSE is an extremely difficult scientific nut to crack.

\section{H. Schellekens}

Diagnostic Center SSDZ,

PO Box 5010,

2600 GA Delft, The Netherlands

SIR - Van Bekkum and Heidt confirm the truth that every cloud has a silver lining in the case of the BSE scare, an opportunity to "dispose of" numbers of chimpanzees surplus to requirements of the AIDS research programme in Europe. You recently reported a similar chimpanzee glut at the US National Institutes of Health (Nature 381, 722; 1996) but in that case the proposed solution (retirement homes) seemed rather more humane than van Bekkum and Heidt's suggestion of "intra-cerebral injection of BSE brain and beef and administration of infected cow brain and meat and perhaps even milk by the cutaneous, intravenous and... oral route".

They are of course right to say that the BSE saga is full of unknowns; neither the relation of the 'new variant' of CreutzfeldtJakob disease to BSE, the relation of BSE to animal feed (or insecticide?) or the extent of risk to the human population is established. However, a couple of other things are well known but, strangely, not yet acted on. The first is that BSE is a direct result of intensive farming methods ('factory farming' of animals) which are generally objectionable on animal-welfare grounds. The second is that these methods have reduced the price of meat and dairy products to the extent that we in the rich countries now damage our health by eating too much of them and damage the environment to feed our habit. So perhaps the lessons of BSE, if read aright, will lead to improvements in both human and animal welfare - another silver lining unnoticed by van Bekkum and Heidt even if they come too late for the hapless chimpanzees?

\section{J. Z. Turner}

Crystallography Department,

Birkbeck College,

London WC1E 7HX, UK

e-mail: j.turner@mail.cryst.bbk.ac.uk 